\title{
Steady-state crack growth in single crystals under Mode I loading
}

\author{
Juul, Kristian Jørgensen; Nielsen, Kim Lau; Niordson, Christian Frithiof
}

Published in:

Journal of the Mechanics and Physics of Solids

Link to article, DOI:

10.1016/j.jmps.2017.01.012

Publication date:

2017

Document Version

Peer reviewed version

Link back to DTU Orbit

Citation $(A P A)$ :

Juul, K. J., Nielsen, K. L., \& Niordson, C. F. (2017). Steady-state crack growth in single crystals under Mode I loading. Journal of the Mechanics and Physics of Solids, 101, 209-222.

https://doi.org/10.1016/j.jmps.2017.01.012

\section{General rights}

Copyright and moral rights for the publications made accessible in the public portal are retained by the authors and/or other copyright owners and it is a condition of accessing publications that users recognise and abide by the legal requirements associated with these rights.

- Users may download and print one copy of any publication from the public portal for the purpose of private study or research.

- You may not further distribute the material or use it for any profit-making activity or commercial gain

- You may freely distribute the URL identifying the publication in the public portal

If you believe that this document breaches copyright please contact us providing details, and we will remove access to the work immediately and investigate your claim. 


\title{
Steady-State Crack Growth in Single Crystals under Mode I Loading
}

\author{
K. J. Juul*, K. L. Nielsen, C. F. Niordson \\ Department of Mechanical Engineering, Solid Mechanics, Technical University of \\ Denmark, DK-2800 Kgs. Lyngby, Denmark
}

\begin{abstract}
The active plastic zone that surrounds the tip of a sharp crack growing under plane strain Mode I loading conditions at a constant velocity in a single crystal is studied. Both the characteristics of the plastic zone and its effect on the macroscopic toughness is investigated in terms of crack tip shielding due to plasticity (quantified by employing the Suo, Shih, and Varias set-up). Three single crystals (FCC, BCC, HCP) are modelled in a steadystate elastic visco-plastic framework, with emphasis on the influence ratesensitivity and crystal structures. Distinct velocity discontinuities at the crack tip predicted by Rice [Rice J.R., 1987. Tensile crack tip fields in elasticideally plastic crystals. Mech. Mater. 6, pp. 317-335] for quasi-static crack growth are confirmed through the numerical simulations and highly refined details are revealed. Through a detailed study, it is demonstrated that the largest shielding effect develops in HCP crystals, while the lowest shielding exists for FCC crystals. Rate-sensitivity is found to affect the plastic zone size, but the characteristics overall remain similar for each individual crystal structure. An increasing rate-sensitivity at low crack velocities monotonically increases the crack tip shielding, whereas the opposite behaviour is observed at high velocities. This observation leads to the existence of a characteristic velocity at which the crack tip shielding becomes independent of the ratesensitivity.
\end{abstract}

Keywords: Quasi-static crack growth, Crystal plasticity, Plastic zones, Shielding effect, Rate-sensitivity

\footnotetext{
*Corresponding author

Email address: krjoju@mek.dtu.dk (K. J. Juul)
}

Preprint submitted to Journal of the Mechanics and Physics of Solids January 23, 2017 


\section{Introduction}

The active plastic zone that surrounds a crack tip has a significant influence on the fracture toughness (a composition of plastic dissipation and the work of separation), and it is the primary condition for obtaining stable crack growth. The near tip plastic zone acts as a shield against the elastic far field, which follows the well-known $\sqrt{r}$-singularity in the stresses, and in this way plasticity increases the toughness of the material both by dissipating energy and by lowering the near tip stress field. The active plastic zone, that surrounds the crack tip, will follow the tip during growth and create a wake of residual plastic strains as the material elastically unloads on the trailing edge. In the regions of unloading, close to the crack face, reversed plastic straining can occur. This results in continued yielding of the material, but in the opposite direction. A wide range of parameters, that describes both the material and the loading, have an influence on the development of the near tip plastic zones, and thus also on the macroscopic fracture toughness. In particular, the strain hardening of the material, governing plastic deformation, is known to influence the energy dissipation and thereby affects the energy required for the crack to advance. Thus, the strain rate hardening, that follows from rate-sensitivity, must be expected to share a similar effect on the shielding and the material toughness. The effect of the viscous behaviour was brought out in e.g. Nielsen and Niordson (2012a) for a Mode I crack travelling at steady-state in an isotropic material. Their study revealed a significant increase in the crack tip shielding for slowly growing cracks compared to a fast growing crack. Moreover, the study of Nielsen and Niordson (2012a) showed that in-between what is characterised as a slowly and a fast growing crack, a velocity leading to the rate-independent toughness can be determined.

Published studies on fracture toughness mainly considers isotropic materials (see e.g. Dean and Hutchinson, 1980; Hui, 1983; Suo et al., 1993; Tvergaard, 1997; Wei and Hutchinson, 1999; Nielsen and Niordson, 2012a). However, single crystals have been in focus due to their brittle to ductile transition temperature (see e.g. Roberts et al., 1993; Tarleton and Roberts, 2009) as-well as their distinct crack tip plastic fields (see e.g. Rice, 1987; Rice

et al., 1990; Ortiz et al., 1992). Both static, quasi-static and dynamic cases ranging over both analytical and numerical calculations have been pursued. 
The effort in these studies has been put on determining and proving the existence of specific characteristics of the material behaviour in the vicinity of the crack tip. In single crystals, the crack tip characteristics reveal themselves as angular sectors separated by either stress or velocity discontinuities (depending on whether the crack is static or growing quasi-statically). The numerical quasi-static case performed by Rice et al. (1990) is based on a traditional Lagrangian framework with a crack growing though a transient phase until steady-state is achieved. Here, accepting a sparse discretization of the domain of interest to make the computations feasible. However, this has been improved significantly in the present study by adopting the steadystate approach by Dean and Hutchinson (1980) which directly brings out the field of interest and allows focusing the computational effort. Moreover, by combining the computational framework with the SSV-model proposed by Suo et al. (1993), a direct comparison of the crack tip shielding for various crystal structures can be conducted in a rigorous manner.

The goal of the present study is to analyse quasi-static crack growth in rate-sensitive single crystals (FCC, BCC, HCP) under Mode I loading. In this way, the study has two parts; I) The first part is an investigation of the characteristics of the plastic zone surrounding the crack tip for the different crystallographic structures. This enables comparison to the work of Rice (1987) and Rice et al. (1990), but also sheds new light on the problem as the true steady-state is obtained within a modified boundary layer framework. The effect of rate-sensitivity on the plastic zones will be brought out; II) The second part of the study investigates the macroscopic crack tip shielding under the assumption that the failure of the material is controlled by cleavage cracking. The analysis of the shielding is based on the SSV-model by Suo et al. (1993), which facilitates an energy based fracture criterion evaluated by the J-integral. The effect of rate-sensitivity is of primary concern as the viscous behaviour significantly influences the plastic field.

The paper is divided into the following sections: The modified boundary value formulation is presented in Section 2, the material model and the numerical formulation are presented in Section 3, validation and results are presented in Section 4, and at last some concluding remarks are given in Section 5. Index notation including Einstein's summation convention is used, and the notation $\left({ }^{\cdot}\right)$ signifies a time derived quantity. 


\section{Problem}

The study is conducted under small scale yielding and treated as quasistatic i.e. the effect of inertia is neglected. A Mode I far field loading is applied on the outer boundary of the discretized material domain (illustrated in Fig. 1) according to the modified boundary layer formulation (Dean and Hutchinson, 1980), whereby the far field loading is controlled by the stress intensity factor $K_{I}$

$$
\sigma_{i j}=\frac{K_{I}}{\sqrt{2 \pi r}} f_{i j}(\theta)
$$

where $r$ and $\theta$ are polar coordinates related to the crack tip position and $f_{i j}(\theta)$ are dimensionless mode functions. By introducing a reference plastic zone size parameter, $R_{0}$, depending on a reference stress intensity factor, $K_{0}$, as

$$
R_{0}=\left(\frac{K_{0}}{\tau_{0}}\right)^{2}, \text { and } \quad K_{0}=\sqrt{\frac{E \Gamma_{t i p}}{1-\nu^{2}}}
$$

the energy release rate at the crack tip, $\Gamma_{t i p}$ (microscopic fracture energy), can be used as a local linear elastic fracture criterion $\left(J_{t i p}=\Gamma_{t i p}\right)$ facilitated by modelling the SSV domain as will be described in Section 3.3. The macroscopic fracture energy, $J_{s s}$, is related to the stress intensity factor $K_{I}$, applied at the boundary (see Fig. 1), through a relation similar to Eq. (2).

The crack growth problem is analysed in the $2 \mathrm{D}$ plane strain steadystate framework suggested by Dean and Hutchinson (1980), whereby the crack propagates at a constant velocity, $\dot{a}$. The numerical procedure iterates directly on the stationary condition where the stress and strain field becomes constant to an observer that follows the crack tip.

The 2D plane strain case is of special interest as these studies allow for detailed experimental investigations (see e.g. Kysar et al., 2005; Dahlberg et al., 2014). In order to analyse a material with a 3D crystallographic structure in a 2D plane strain setting, it is necessary to invoke effective slip systems by combining the out-of-plane slip systems to equivalent in plane slip systems (particularly for the FCC and BCC structures). A description of the effective slip systems can be found in Section 2.1.

The single crystal structures investigated belongs to the three families most commonly found in metals; the face centered cubic (FCC), the body 
centered cubic $(\mathrm{BCC})$, and the hexagonal close packed (HCP). It should be mentioned, however, that cleavage cracking is not equally likely in all crystal structures. It is rather unlikely for cleavage to occur in an FCC crystal since ample slip systems for ductile behaviour exist at all temperatures for this particular crystal structure. At low temperatures, cleavage can occur in BCC crystal as only a limited number of active slip systems exist. Cleavage is also likely to be observed in HCP crystals as few slip systems are active (Anderson, 2005).

Since the material model is based on an elastic visco-plasticity theory, a definition of the active plastic zone that engulfs the crack tip is required. The quantity utilized in the present work is based on the absolute value of the accumulated slip rate, $\dot{\Lambda}=\sum_{\alpha}\left|\dot{\gamma}^{(\alpha)}\right|$, as suggested by Rice et al. (1990). The material properties adopted for the study can be found in Tab. 1 .

\subsection{Effective Slip Systems}

To create a 2D plane strain deformation of the single crystals specific orientations are required, such that any out of plane action from one slip system are cancelled by one or more other slip systems (see e.g. Rice, 1987; Kysar et al., 2005; Niordson and Kysar, 2014). By considering the symmetry plane (101) for plane strain deformation in FCC and BCC crystals, crystallographic slip systems can be combined pairwise into equivalent so-called effective slip systems where the pair is activated equally with respect to the slip such that out-of-plane actions cancel out. In FCC crystals, three such effective slip systems exist which are denoted $(\alpha)$, while the two crystallographic slip systems combined into each effective slip system are denoted $(\alpha a)$ and $(\alpha b)$. This can be envisioned by for example having an equal amount of slip on the

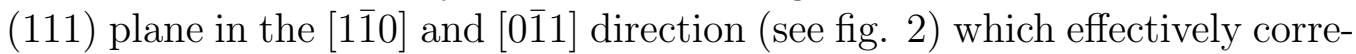
sponds to slip in the [1리] direction. For BCC crystals, only one effective slip system is constructed as the other crystallographic slip systems are already in the plane of interest. This effective slip system is constructed from Fig. 2

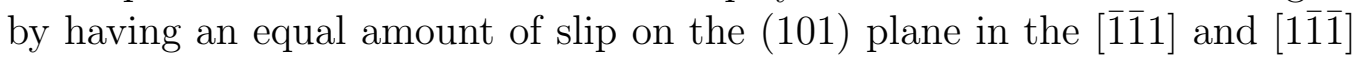
direction corresponding to slip in the [020]0] direction. For an HCP crystal, oriented such that the basal plane (0001) is in the plane of the deformation, no effective slip systems are needed and existing prismatic crystallographic slip systems are modelled directly. The parameters and method for determining the effective slip systems are adopted from Rice (1987) and Niordson and Kysar (2014). Table 2 presents the individual crystallographic slip systems, the corresponding effective slip systems, and the crack orientation used in the 
analysis (also illustrated in Fig. 3). In Tab. 2, $\beta^{(\alpha)}$ is the effective parameter, describing the relation between the slip on the crystallographic slip systems and the corresponding effective slip system, ensuring equivalent deformation. The parameter $\lambda^{(\alpha)}$, gives the relation between the resolved shear stress on the crystallographic slip systems and the corresponding effective slip system. The scaling of the resolved shear stress and slip, when utilizing the effective systems, can thereby be expressed as the initial slip resistance, $\tau_{0}$, and the reference strain rate, $\dot{\gamma}_{0}$, multiplied by $\lambda^{(\alpha)}$ and $\beta^{(\alpha)}$ (see Tab. 2 for specific values), respectively. Hence, each effective slip system will have its own value of the slip resistance and reference strain rate according to

$$
\tau_{0}^{(\alpha)}=\lambda^{(\alpha)} \tau_{0}, \quad \text { and } \quad \dot{\gamma}_{0}^{(\alpha)}=\beta^{(\alpha)} \dot{\gamma}_{0} .
$$

As will be shown from the numerical results (see Section 4), the added corrections to the individual effective slip systems severely influence the appearance of the plastic zone that travels with the propagating crack tip.

\section{Numerical Framework}

\subsection{Rate-sensitive Material Model}

A small strain formulation is employed where the total strain, $\varepsilon_{i j}$, is determined from the displacement, such that $\varepsilon_{i j}=\left(u_{i, j}+u_{j, i}\right) / 2$, which is decomposed into an elastic part, $\varepsilon_{i j}^{e}$, and a plastic part, $\varepsilon_{i j}^{p}\left(\varepsilon_{i j}=\varepsilon_{i j}^{e}+\varepsilon_{i j}^{p}\right)$. Based on the strain field, the stress field is determined from the elastic relationship; $\sigma_{i j}=\mathscr{L}_{i j k l}\left(\varepsilon_{k l}-\varepsilon_{k l}^{p}\right)$, where $\mathscr{L}_{i j k l}$ is the elastic stiffness tensor. The total plastic strain rate, $\dot{\varepsilon}_{i j}^{p}$, in a single crystal is determined by summation over all slip systems, $\alpha$, according to

$$
\dot{\varepsilon}_{i j}^{p}=\sum_{\alpha} \dot{\gamma}^{(\alpha)} P_{i j}^{(\alpha)}, \quad P_{i j}^{(\alpha)}=\frac{1}{2}\left(s_{i}^{(\alpha)} m_{j}^{(\alpha)}+m_{i}^{(\alpha)} s_{j}^{(\alpha)}\right)
$$

where $P_{i j}^{(\alpha)}$ is the Schmid orientation tensor, $\dot{\gamma}^{(\alpha)}$ is the slip rate on a specific slip system, and $s_{i}^{(\alpha)}$ and $m_{i}^{(\alpha)}$ are two unit vectors defining the slip direction and the slip plane normal, respectively (see Fig. 3). The slip rate on each slip system, $\alpha$, is based on the visco-plastic power law slip rate relation proposed 
by Hutchinson (1976)

$$
\dot{\gamma}^{(\alpha)}=\dot{\gamma}_{0} \operatorname{sgn}\left(\tau^{(\alpha)}\right)\left(\frac{\left|\tau^{(\alpha)}\right|}{g^{(\alpha)}}\right)^{1 / m}
$$

where $\tau^{(\alpha)}=\sigma_{i j} m_{i}^{(\alpha)} s_{j}^{(\alpha)}$ is the resolved shear stress and $g^{(\alpha)}$ is the slip resistance evolving during plastic straining (the elasticity is assumed isotropic i.e. effects of elastic anisotropy are ignored). The relationship between the slip resistance, $g^{(\alpha)}$, and the plastic straining is given by the power law relation; $g^{(\alpha)}=\tau_{0}\left(1+G\left|\gamma_{a c c}^{(\alpha)}\right| / \tau_{0}\right)^{N}$, where $G$ is the shear modulus and $\gamma_{a c c}^{(\alpha)}=\int\left|\dot{\gamma}^{(\alpha)}\right| \mathrm{d} t$ is the accumulated slip on slip system $(\alpha)$. It is evident from the slip resistance function that only self-hardening is considered in this study i.e. the hardening on a slip system is solely a result of slip on the system itself. Latent hardening, where slip on one system can affect another system, is neglected for simplicity. Furthermore, the role of twinning, which may be of importance in some metals (e.g. Mg and TiAl alloys), is not treated, however, for a more comprehensive study of these specific alloys the effect should be included.

According to Eq. (5), the rate-sensitivity of the material response increases as the rate-sensitivity exponent, $m$, increases and vice versa. This also implies that for $m \rightarrow 0$, the constitutive material model approach the response of the rate-independent material.

\subsection{Steady-State Approach}

The present study analyses the plastic zone that surrounds the tip of a sharp cleavage crack, growing at constant velocity, to bring out its effect on the material toughness (the shielding ratio). In doing so, a steady-state framework is an ideal choice as it directly brings out details on the crack tip conditions in a frame translating with the moving crack tip. In addition, the numerics also have the benefit of avoiding to explicitly model the transient period from crack initiation to steady-state growth. The steady-state finite element model employed in the present study is based on the early work of Dean and Hutchinson (1980). The steady-state condition for a continuously growing crack is described as the condition where the field quantities that surrounds the crack tip remains unchanged relative to an observer located at the crack tip. The steady-state condition states that any time derived quantity, $\dot{f}$, in the constitutive model can be related to a spatial derivative 
through the velocity, $\dot{a}$, along a streamline, according to the relation $\dot{f}=$ $-\dot{a} \partial f / \partial x_{1}$ (the minus sign is due to material flow in the negative $x_{1}$-direction as illustrated in Fig. 1). Thus, any incremental quantity at a given material point $\left(x_{1}^{*}, x_{2}^{*}\right)$, can be evaluated by integrating along a streamline, starting upstream in the elastic zone well in front of the crack tip $\left(x_{1}^{0}, x_{2}^{*}\right)$ and ending at the point of interest downstream $\left(x_{1}^{*}, x_{2}^{*}\right)$ (see e.g. Juul et al., 2017). The point of interest $\left(x_{1}^{*}, x_{2}^{*}\right)$ will then contain the load history of all upstream points. The streamline integration procedure is performed with a classical forward Euler integration scheme.

In the adopted steady-state framework, the displacement field, $u_{i}$, is determined based on the conventional principle of virtual work (PWV) for quasi-static problems

$$
\int_{V} \mathscr{L}_{i j k l} \varepsilon_{k l} \delta \varepsilon_{i j} \mathrm{~d} V=\int_{S} T_{i} \delta u_{i} \mathrm{~d} S+\int_{V} \mathscr{L}_{i j k l} \varepsilon_{k l}^{p} \delta \varepsilon_{i j} \mathrm{~d} V
$$

where $T_{i}=\sigma_{i j} n_{j}$ is the surface traction. The volume analysed is denoted $V$, and $S$ is the bounding surface, with $n_{j}$ denoting the unit outward normal vector.

The implementation of the virtual work principle follow a procedure similar to the one suggested by Nielsen and Niordson (2012a) for an isotropic visco-plastic steady-state model with the exception that kinematic relations for a single crystalline material is employed here. This implementation procedure also slightly deviate from the work of Dean and Hutchinson (1980), as this is a time dependent model. For a time dependent model, the history dependence will enter through the plastic strain instead of through the stresses as in the original procedure (the plastic strains are streamline integrated rather than the stresses). The virtual work principle in Eq. (6) has been discretized using a quadratic 8-node isoparametric element with reduced Gauss integration $(2 \times 2$ Gauss points). The pseudo algorithm for the rate-sensitive steady-state procedure in single crystals is as follows ( $n$ refers to the iterative step):

1. The plastic strain from the previous iteration, $\varepsilon_{i j}^{p(n-1)}$, is used to determine the current displacement field, $u_{i}^{(n)}$, from the principle of virtual work in Eq. (6).

2. The total strain, $\varepsilon_{i j}^{(n)}$, is determined based on the displacement field, $u_{i}^{(n)}$. 
3. The slip and plastic strain fields are determined by the streamline integration procedure.

(a) First the spatial derivative of the slip (on the individual slip planes) and total plastic strains are determined by utilizing the steadystate relation $\left(\partial f / \partial x_{1}=-\dot{f} / \dot{a}\right)$

$$
\begin{aligned}
\frac{\partial \gamma^{(\alpha)}}{\partial x_{1}} & =-\frac{\dot{\gamma}_{0}}{\dot{a}} \operatorname{sgn}\left(\tau^{(\alpha)}\right)\left(\frac{\left|\tau^{(\alpha)}\right|}{g^{(\alpha)}}\right)^{1 / m} \\
\frac{\partial \varepsilon_{i j}^{p}}{\partial x_{1}} & =\sum_{\alpha} \frac{\partial \gamma^{(\alpha)}}{\partial x_{1}} P_{i j}^{(\alpha)}
\end{aligned}
$$

(b) Secondly, the current slip $\gamma^{(\alpha)(n)}$ on each system and the current plastic strains, $\varepsilon_{i j}^{p(n)}$, are determined from the spatial derivatives by performing the streamline integration

$$
\gamma^{(\alpha)(n)}=\int_{x_{1}^{0}}^{x_{1}^{*}} \frac{\partial \gamma^{(\alpha)}}{\partial x_{1}} \mathrm{~d} x_{1}, \quad \text { and } \quad \varepsilon_{i j}^{p(n)}=\int_{x_{1}^{0}}^{x_{1}^{*}} \frac{\partial \varepsilon_{i j}^{(p)}}{\partial x_{1}} \mathrm{~d} x_{1}
$$

4. The current stress field $\sigma_{i j}^{(n)}$ is determined using the elastic constitutive relation.

5. Step 1 through 4 is repeated by feeding the newly found plastic strain into the right hand side of the PWV in Eq. (6) until convergence is obtained.

The iterative procedure is initiated by using the purely elastic solution to the problem i.e. $\gamma^{(\alpha)}=0$ for the first step. The numerical stability of the steady-state algorithm has been found to be very sensitive to various parameters, and especially for low rate-sensitivity exponents, $m$, difficulties with obtaining convergence is encountered. In order to improve the numerical stability of the algorithm, changes have been made to the original procedure by Dean and Hutchinson (1980). The changes follow the suggestion by Niordson (2001) and Nielsen and Niordson (2012a), where subincrement between Gauss points are introduced in the streamline procedure.

The steady-state model for single crystals has proven difficult to validate as limited literature exists on the topic. Thus, besides comparing to the analytical and numerical results of Rice (1987); Rice et al. (1990), the model has 
been compared to a strict plane strain isotropic model developed separately. By systematically adding more slip systems a field matching the isotropic model prediction was achieved.

\subsection{The SSV model}

Suo et al. (1993) presented a framework (the SSV-model) for cleavage cracking surrounded by pre-existing dislocations. This model has been chosen as it offers a simple and very robust method to evaluate the crack tip shielding. The SSV-model is based on the assumption that no dislocations are emitted from the crack front. This statement requires that the dislocation spacing is much larger than the lattice constant, whereby the probability for a pre-existing dislocation to blunt a major part of the crack tip is minor. When no dislocations are emitted from the crack tip, the crack will propagate by atomic separation, and thereby remain infinity sharp. Within this region, where no dislocations are present, the material will therefore behave elastically. When relating this to the numerical steady-state procedure, this means that the crack is embedded within a thin material strip of height $2 D$ (see Fig. 1), which behaves elastically (the influence of the SSV domain geometry is investigated in Tvergaard, 1997). As the crack tip is embedded in an elastic zone, linear elastic fracture mechanics applies, and the energy release rate can be evaluated by the J-integral following the procedure of Shih et al. (1986). When applying the J-integral (within the elastic SSV domain), the corresponding fracture criterion is $J_{t i p}=\Gamma_{t i p}$, where $\Gamma_{t i p}$ denotes the energy release rate required for the crack to advance. It should be mentioned that the SSV-model is not valid if the length of the separation zone at the crack tip becomes comparable to the magnitude of the elastic strip, $D$. Thus, the SSV-model is only valid for materials in which fracture is dominated by cleavage or atomic separation (see detailed discussion in e.g. Wei and Hutchinson, 1999). The height of the SSV domain can either be regarded as a material fitting parameter (Suo et al., 1993) or it can be estimated using dislocation theory (Beltz et al., 1996; Lipkin et al., 1996).

Based on the problem presented in Section 2, the crack tip shielding ratio, $J_{s s} / J_{t i p}$, is governed by the dimensional analysis conducted by Wei and Hutchinson (1999), where $J_{s s}$ is the remotely applied energy release rate. This dimensional analysis states that the shielding ratio at steady- 
state is given by

$$
\frac{J_{s s}}{J_{t i p}}=F\left(\frac{\dot{a}}{R_{0} \dot{\gamma}_{0}}, \frac{R_{0}}{D}, \frac{\tau_{0}}{G}, N, m, \nu\right)
$$

where the quantity $\dot{a} /\left(R_{0} \dot{\gamma}_{0}\right)$ will be denoted $\zeta$, to represent a dimensionless velocity. The parameter groups identified in Eq. (10) are therefore of key interest in developing a parametric understanding of crack growth in single crystals.

\section{Results}

The mesh employed in the model contains 102400 elements and is gradually scaled in two directions to obtain a very fine mesh in the vicinity of the crack tip where details are required. Approximately 19000 of the 102400 elements are concentrated within a region comparable to the plastic zone size in order to give a detailed solution.

\subsection{Active Plastic Zones in Single Crystals}

The first part of the results concerns the active plastic zone in the vicinity of the crack tip. These fields have previously been studied by Rice (1987) and Rice et al. (1990) for quasi-static crack growth, both analytically and numerically. In Rice (1987), analytical results showed that distinct zones, involving unloading and reloading to the yield point, takes place in the vicinity of the crack tip (see Fig. 4). These zones are seen as angular sectors which are separated by discontinuities in the velocity field at very specific locations related to the crystal orientation. The angles separating the sectors are characterized by being either perpendicular or parallel to the slip systems. The original analysis by Rice (1987) relies on perfectly plastic material behaviour $(N \rightarrow 0)$, in the rate-independent limit $(m \rightarrow 0)$, under Mode I loading condition. Rice (1987) presented analytical results for crack growth in the [101] direction with the crack plane orthogonal to the [010] direction, for both the FCC and BCC crystal structures. These crystal structures prove to have the same angular locations of the discontinuities since the effective slip systems in FCC and BCC crystal structures are perpendicular to each other.

In a later study, Rice et al. (1990) conducted a numerical investigation of the FCC structure in a quasi-static setting, validating the analytical results, by analysing the near tip plastic zone of a propagating crack. Direct comparison to the numerical results of Rice et al. (1990), is unfortunately not 
possible as the propagation velocity in that particular analysis is unknown. Despite this, the results can still be compared qualitatively. To improve on these early results and bring out the effect of rate-sensitivity, the active plastic zones for steady-state growth is presented in Figs. 5, 6, and 7 for FCC, $\mathrm{BCC}$, and HCP crystal structures, respectively. Here, the zones are shown for different rate-sensitivity exponents, $m$, and a constant growth velocity of $\zeta=\dot{a} /\left(R_{0} \dot{\gamma}_{0}\right)=1000$. The criterion for plasticity in the vicinity of the crack tip has been adopted from Rice et al. (1990), and it is based on the accumulated slip $\left(\dot{\Lambda}=\sum_{\alpha}\left|\dot{\gamma}^{\alpha}\right|\right)$. However, it should be mentioned that in the results of Rice et al. (1990), $\dot{\Lambda}$ is normalized by $\tau_{0} / G$, whereas here it is normalized by $\zeta \dot{\gamma}_{0} \tau_{0} / G$ in order to obtain non-dimensionality and comparable fields across a large velocity span.

In contrast to Rice et al. (1990), who relied on a crack growing transiently until it reaches steady-state, the results in Figs. 5-7 provide detailed steadystate results for the plastic zone. Moreover, the purpose build framework allows the computational effort to be focused on the crack tip such that highly refined discretization can be employed. Comparing the findings of Rice et al. (1990) (numerical results for the FCC crystal), to the results presented in Fig. 5a reveals a striking match. Similar features, consisting of two large active plastic features, (B) and (D), and a plastic wake, A), are observed. The expected velocity discontinuities illustrated by the white regions (zones of largely concentrated plastic straining), correspond to the prediction by Rice (1987) (see Fig. 4) with discontinuities at $54.7^{\circ}$ and $125.3^{\circ}$ and moreover, the size of the plastic features are of the same order of magnitude. The plastic feature denoted (B) is, however, somewhat longer compared to feature (D) in the present results. One possible explanation of this could be that the solution in Rice et al. (1990), remains to fully reach the steady-state. In their corresponding field for a stationary crack, Rice et al. (1990) demonstrates a much different appearance of features (B) and (D) (where feature (B) is almost absent), thus the features will have to evolve significantly and become constant before the steady-state is reached. Another significant difference between the two studies is the level of refinement as the adopted steady-state approach allows focusing the discretization. E.g. both the feature (C) and protrusion on the leading edge of feature (D) (vaguely visible in Rice et al., 1990) stands out very clearly. By increasing the ratesensitivity (see Fig. 5b), the active plastic zones increases in size, however, their characteristics remain similar, with the exception that the inclination of 
feature (B) seems to be diminishing with increasing rate-sensitivity exponent, $m$ (both for slowly and fast growing cracks).

Figure 6 presents similar, but new, results for the BCC crystal structure. Nowhere is a numerical comparison basis found, but according to Rice (1987), identical regions and velocity discontinuities, as for the FCC crystal, should be observed in the BCC crystal. This is confirmed in Fig. 6a for low ratesensitivity exponent, $m$. Comparing the active plastic region for the FCC and BCC cases reveal large similarities, however, the two active plastic features, (B) and (D), are slightly larger for the BCC structure, while the wake, A), is approximately the same size. The difference in magnitude can be explained by the effective slip systems found in Tab. 2. Only the $90^{\circ}$ plane in a BCC crystal is an effective plane which has higher effective resistance to slip due to the scaling from Eq. (3). On the other hand, all planes for the FCC crystal are effective planes and thereby get a higher effective resistance to slip, resulting in a smaller plastic zone. Besides the difference in magnitude, the development of the small active plastic feature at (C) is not seen for the BCC crystal structure at low rate-sensitivity. However, at larger ratesensitivity, this feature becomes evident and a larger similarity to results for the FCC crystal structure shows (compare Figs. 5b and 6b).

Lastly, the results for an HCP crystal structure is presented in Fig. 7. The results for the HCP crystal show a very different magnitude of the active plastic zones compared to both the FCC and BCC crystals. Comparing the results for low rate-sensitivity exponent, $m$, in Fig. 7a to the corresponding FCC crystal results (Fig. 5a), the feature (C) has now become much more dominant and the wake, (A), has also increased significantly in magnitude. As for the difference between the FCC and BCC crystals, the change in the plastic zone for the HCP crystal is tied to the corrections of the slip systems according to Eq. (3) (or the lack hereof). The HCP crystal structure has three active slip systems in the 2D plane meaning that creating effective systems are not needed and thus no corrections on the slip systems are imposed. Unfortunately, analytical results are yet to be developed for the HCP crystal structure, and a basis for comparison is missing. However, from the findings in Fig. 7a it is seen that the location of the velocity discontinuities obeys the conditions of being either perpendicular or parallel to the slip systems (as for both FCC and BCC crystals). When comparing to the discontinuities of the FCC and BCC crystals, an additional discontinuity is seen at feature (C), while the features (B) and (D) are closer together (smaller angle 
between discontinuities) due to the $60^{\circ}$ angle between active planes in the HCP crystal.

By increasing the rate-sensitivity exponent, $m$, the active plastic zones grow, just as for FCC and BCC crystals. However, due to the existence of the more significant plastic feature (C), the three zones which stay disjunct for low rate-sensitivity now merge into one (the same tendency is expected for FCC and BCC crystals, however, a larger rate-sensitivity exponent, $m$, would be required). Common for all crystals are that changes in the velocity influence the magnitude of the plastic zone, but the proportions between the individual features remain largely unchanged.

\subsection{Crack Tip Shielding Ratio in Single Crystals}

The SSV-model is now introduced to investigate the shielding effect of the plastic zones for the individual crystal structures. The following studies are conducted under what is referred to as fast and slow crack growth, where the dimensionless crack velocity is $\zeta=1000$ and $\zeta=10$, respectively (recall that $\left.\zeta=\dot{a} /\left(R_{0} \dot{\gamma}_{0}\right)\right)$. It should be noted that the fast growing crack is not reaching velocities where dynamic effects become important and thus it can still be handled as quasi-static crack growth where inertia effects are neglected.

Figure 8 presents the shielding ratio as a function of the height of the elastic (dislocation free) SSV-region for both a slowly (Fig. 8a) and a fast (Fig. 8b) growing crack under Mode I loading. Here, displaying results for the both FCC, BCC, and HCP crystal structures. Common for both the fast and slowly growing crack, in all crystal structures, is the increase in crack tip shielding as the SSV region becomes thinner $\left(R_{0} / D\right.$ increases $)$. Comparing the three different crystal structures it is found that the largest shielding ratio occurs for HCP crystals, while the lowest is found for FCC crystals. This is in good agreement with the correction parameters stated in Tab. 2, where the FCC crystal structure will exhibit the largest resistance against plastic deformation and thereby the smallest plastic zone to shield the crack tip. In contrast, the HCP crystal has the lowest resistance (no correction imposed, as effective systems are not required), and thereby a large plastic zone, that gives rise to a large shielding effect (consistent with previous observations in Figs. 5, 6, and 7). The BCC crystal structure has only one effective slip system, providing additional resistance, and therefore exhibits a crack tip shielding between the FCC and HCP crystal.

The effect of varying the hardening exponent, $N$, for a constant ratesensitivity exponent, $m$, is brought out in Fig. 9. Here, a limited difference 
between the slow and fast cracks is found since the rate-sensitivity exponent, $m$, is maintained fairly low. Regardless of the crack velocity, the same tendency as previously observed, regarding the highest shielding ratio for $\mathrm{HCP}$ crystals and the lowest for FCC crystals, still holds. For both the slow (Fig. 9a) and the fast crack (Fig. 9b) a decreasing hardening exponent, $N$, results in an increasing crack tip shielding due to more plastic deformation.

Another interesting observation, when comparing the fast and slowly growing cracks, regardless of the crystal structure, is the influence of the rate-sensitivity on the shielding ratio. For the slowly growing crack (Fig. $8 a$ ), the shielding is monotonically increasing with increasing rate-sensitivity exponent, $m$, while the opposite effect of a monotonically decreasing shielding is seen for the fast growing crack (Fig. 8b). The monotonic increase (decrease), for the low (high) velocity, naturally implies that the lines for different rate-sensitivities must intersect at one uniquely defined velocity (in accordance with the findings of Nielsen and Niordson (2012a) for isotropic materials). This behaviour is related to the rate dependency introduced through Eq (5) and it is not specific to isotropic nor single crystalline materials. The phenomenon can be explained by the same statement as Nielsen and Niordson (2012a) put forward which is based on the time aspect of the rate-sensitive model. Stress build-up or relaxation of the material occurs in the vicinity of the crack tip depending on the combination of velocity and rate-sensitivity. At low velocities, the material is given time to relax during the crack growth resulting in larger plastic strains i.e. more plastic dissipation and thus a larger shielding. In the opposite case where high velocities prevail, the relaxation is limited and leads to higher stresses (less plasticity) in the vicinity of the crack tip and thus lower shielding. This behaviour is largely dependent on the rate-sensitivity exponent, $m$, which will make the effect more or less pronounced.

To investigate the phenomena of a characteristic velocity, the uniquely defined intersection of the curves is further investigated in Fig. 10, where the shielding ratio is displayed as function of the dimensionless velocity, $\zeta=\dot{a} /\left(R_{0} \dot{\gamma}_{0}\right)$, for all three crystal structures. Clearly, it is common for all that a characteristic crack growth velocity exists (for fixed height of the SSV domain, $R_{0} / D$, and hardening exponent, $N$ ), at which the shielding becomes independent of the rate-sensitivity exponent, $m$. This despite that the plastic zones may vary for different rate-sensitivities at this velocity. The existence of this characteristic velocity, however, opens up for the possibility of studying the rate-independent response using a rate-dependent model (see 
also discussion in Nielsen et al., 2012b).

From Fig. 10 it is noticed that the characteristic velocity for the $\mathrm{BCC}$ and HCP crystals are very close, with the BCC crystal having a slightly larger value, whereas the characteristic velocity for the FCC crystal is significantly larger. Moreover, it is worth mentioning that the characteristic velocity for the HCP crystal in Fig. 10a (low hardening), is slightly off compared to the intersection in Fig. 10b (high hardening). A less distinct intersection of the curves is generally observed when the amount of plasticity is increasing in the problem as the large plastic zone tends to give difficulties in obtaining convergence.

\section{Concluding Remarks}

The active plastic zone that travels with a steadily growing crack in various single crystals have been analysed in detail. The crack is modelled in a steady-state framework where it is subject to Mode I loading in a ratesensitive material setting. In accordance with Rice (1987), distinct sectors that divide the domain near the crack tip have been identified for the three most commonly encountered crystal structures in metals (FCC, BCC, and $\mathrm{HCP}$ ). The size and shape of the plastic zone significantly affect the macroscopic fracture toughness of the material (the crack tip shielding ratio) as investigated by applying the SSV model (Suo et al., 1993). The main focus is on the effect of rate-sensitivity as-well as the effect of changing the crystal structures. The key findings are:

- Numerical simulation of single crystal indeed reveal discontinuities corresponding to the analytical results of Rice (1987), which are either perpendicular or parallel to the slip systems. Comparing results for low and high rate-sensitivity reveals that the active plastic zone changes in size, but the characteristics remain largely unchanged. The active plastic zone is very similar for the FCC and BCC crystal structures, while the HCP crystal structure differs substantially. This is linked to the scaling factors that affect plastic flow on the effective slip systems in the adopted 2D plane strain setting. The magnitude of the active plastic zone is smallest for the FCC crystal and largest for the HCP crystal.

- The shielding ratio is smallest for the FCC crystal and largest for the HCP crystal, consistent with the magnitude of the plastic zones for 
the two different crystal structures. Generally, the shielding ratio is observed to increase with $R_{0} / D$. As $R_{0} / D$ increases, the SSV domain decreases in height, which in turn result in more plastic dissipation that contributes to the crack tip shielding. The shielding ratio also increases for a decreasing hardening exponent, $N$, by the same argument, namely that the plastic dissipation is increasing.

- At low velocities, increasing rate-sensitivity leads to a monotonically increasing crack tip shielding ratio, whereas the opposite is observed for high velocities. This monotonically increase/decrease in the response lead to the finding of a characteristic velocity at which the shielding ratio becomes independent of the rate-sensitivity. The BCC and HCP crystal structures are found to have similar characteristic velocities (the BCC structure is slightly larger than the HCP structure), whereas the FCC crystal structure has a significantly larger characteristic velocity.

\section{Acknowledgement}

The work is financially supported by The Danish Council for Independent Research in the project "New Advances in Steady-State Engineering Techniques", grant DFF-4184-00122. Ph.D Chris Valentin Nielsen, DTU Mechanical Engineering, is greatly acknowledged for his parallelization of the skyline solver module (Nielsen et al., 2012). 


\section{References}

Anderson, T. L., 2005. Fracture Mechanics: Fundamentals and Applications, Third Edition. Taylor and Francis.

Beltz, G. E., Rice, J. R., Shih, C. F., Xia, L., 1996. A self-consistent model for cleavage in the presence of plastic flow. Acta Materialia 44, 3943-3954.

Dahlberg, C. F. O., Saito, Y., Öztop, M. S., Kysar, J. W., 2014. Geometrically necessary dislocation density measurements associated with different angles of indentations. Int. J. Plas. 54, 81-95.

Dean, R. H., Hutchinson, J. W., 1980. Quasi-static steady crack growth in small-scale yielding. Fracture Mechanics: Twelfth Conference, ASTM STP700, American Society for Testing and Materials, 383-405.

Hui, C. Y., 1983. Steady-state crack growth in elastic power-law creeping materials. In: elastic-plastic fracture: second symposium. Inelastic crack analysis, ASTM STP803, vol. 1, American Society for Testing and Materials, 573-93.

Hutchinson, J. W., 1976. Bounds and self-consistent estimates for creep of polycrystalline materials. Proc. R. Soc. Lond. A 348, 101-127.

Juul, K. J., Nielsen, K. L., Niordson, C. F., 2017. Steady-state numerical modeling of size effects in micron scale wire drawing. J. Manuf. Process. $25,163-171$.

Kysar, J. W., Gan, Y. X., Mendez-Arzuza, G., 2005. Cylindrical void in a rigid-ideally plastic single crystal. Part I: Anisotropic slip line theory solution for face-centered cubic crystals. Int. J. Plas. 21, 1481-1520.

Lipkin, D. M., Clarke, D. R., Beltz, G. E., 1996. A strain-gradient model of cleavage fracture in plastically deforming materials. Acta Materialia 44, 4051-4058.

Nielsen, C. V., Zhang, W., Alves, L. M., Bay, N., Martins, P. A. F., 2012. Modeling of Thermo-Electro-Mechanical Manufacturing Processes with Applications in Metal Forming and Resistance Welding, First Edition. Springer. 
Nielsen, K. L., Niordson, C. F., 2012a. Rate sensitivity of mixed mode interface toughness of dissimilar metallic materials: Studied at steady state. Int. J. Solids Struct. 49, 576-583.

Nielsen, K. L., Niordson, C. F., Hutchinson, J. W., 2012b. Strain gradient effects on steady-state crack growth in rate-dependent materials. Eng. Fract. Mech. 96, 61-71.

Niordson, C. F., 2001. Analysis of steady-state ductile crack growth along a laser weld. Int. J. Frac. 111, 53-69.

Niordson, C. F., Kysar, J. W., 2014. Computational strain gradient crystal plasticity. J. Mech. Phys. Solids 62, 31-47.

Ortiz, M., Mohan, R., Shih, C. F., 1992. An analysis of cracks in ductile single crystals-II. Mode I loading. J. Mech. Phys. Solids 40, 315-337.

Rice, J. R., 1987. Tensile crack tip fields in elastic-ideally plastic crystals. Mech. Mater. 6, 317-335.

Rice, J. R., Hawk, D. E., Asaro, R. J., 1990. Crack tip fields in ductile crystals. Int. J. Frac. 42, 301-322.

Roberts, S. G., Hirsch, P. B., Booth, A. S., Ellis, M., Serbena, F. C., 1993. Dislocations, cracks and brittleness in single crystals. Phys. Scripta T49, 420-426.

Shih, C. F., Moran, B., Nakamura, T., 1986. Energy release rate along a three-dimensional crack front in a thermally stressed body. Int. J. Frac. 30, 79-102.

Suo, Z., Shih, C. F., Varias, A. G., 1993. A theory for cleavage cracking in the presence of plastic flow. Acta Metall Mater 41, 1551-7.

Tarleton, E., Roberts, S. G., 2009. Dislocation dynamic modelling of the brittle-ductile transition in tungsten. Phil. Mag. 89, 2759-2769.

Tvergaard, V., 1997. Cleavage crack growth resistance due to plastic flow around a near-tip dislocation-free region. J. Mech. Phys. Solids 45, $1007-$ 23.

Wei, Y., Hutchinson, J. W., 1999. Models of interface separation accompanied by plastic dissipation at multiple scales. Int. J. Frac. 95, 1-17. 


\section{List of Figures}

1 Mode I crack growth at steady-state in rate-sensitive crystal plastic material. The SSV domain provide an elastic strip embedded in the steady-state domain (SS domain). The crack is loaded with an elastic $K_{I}$ far field. . . . . . . . . . . . . . . 22

2 FCC and BCC crystal structure with the crack front along the [101] direction in the (010) crack plane (Rice, 1987) . . . . . . . 23

3 Mode I crack growth in single crystal with three and four Miller indices representing FCC/BCC and HCP, respectively. The sharp crack front is along the $[10 \overline{1}] /[0001]$ direction and the three effective slip systems are oriented as shown for FCC, BCC and HCP crystal structures. . . . . . . . . . . . . . . . . 24

4 Velocity discontinuities and angle of secondary plastic zone at a steadily moving crack tip creating angular sectors for FCC and BCC crystals according to Rice (1987). . . . . . . . . . . 25

5 Accumulated slip rate, $\dot{\Lambda}$, for steady-state crack growth in perfectly plastic single crystal showing the plastic region, $\dot{\Lambda} G /\left(\zeta \dot{\gamma}_{0} \tau_{0}\right) \geq$ 1, (black region) and a region of highly concentrated plastic straining, $\dot{\Lambda} G /\left(\zeta \dot{\gamma}_{0} \tau_{0}\right) \geq 2000$, (white region) in an FCC crystal for constant crack velocity $\zeta=1000$ with (a) $m=0.01$, and (b) $m=0.05 \ldots \ldots \ldots$. . . . . . . . . . . . 26

6 Accumulated slip rate, $\dot{\Lambda}$, for steady-state crack growth in perfectly plastic single crystal showing the plastic region, $\dot{\Lambda} G /\left(\zeta \dot{\gamma}_{0} \tau_{0}\right) \geq$ 1, (black region) and a region of highly concentrated plastic straining, $\dot{\Lambda} G /\left(\zeta \dot{\gamma}_{0} \tau_{0}\right) \geq 2000$, (white region) in a BCC crystal for constant crack velocity $\zeta=1000$ with (a) $m=0.01$, and

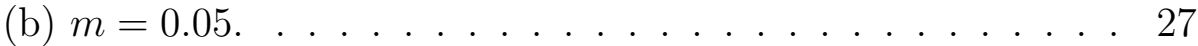

7 Accumulated slip rate, $\dot{\Lambda}$, for steady-state crack growth in perfectly plastic single crystal showing the plastic region, $\dot{\Lambda} G /\left(\zeta \dot{\gamma}_{0} \tau_{0}\right) \geq$ 1, (black region) and a region of highly concentrated plastic straining, $\dot{\Lambda} G /\left(\zeta \dot{\gamma}_{0} \tau_{0}\right) \geq 2000$, (white region) in an HCP crystal for constant crack velocity $\zeta=1000$ with (a) $m=0.01$,

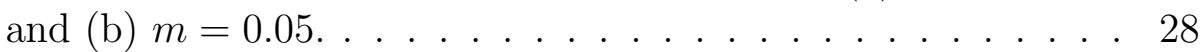

8 Crack tip shielding ratio vs. inverse of dislocation free region (SSV), $D$, for single crystal with parameters; $N=0.10$ and velocity (a) $\zeta=10$, and (b) $\zeta=1000 \ldots . . . . . .29$ 
591

9 Crack tip shielding ratio vs. inverse of dislocation free region (SSV), $D$, for single crystal with parameters; $m=0.01$ and velocity (a) $\zeta=10$, and (b) $\zeta=1000 \ldots \ldots . \ldots . \ldots 30$

10 Crack tip shielding ratio as function of velocity for constant SSV domain size $R_{0} / D=80$ with hardening exponent (a) $N=0.10$, and (b) $N=0.20 \ldots \ldots \ldots \ldots \ldots$ 


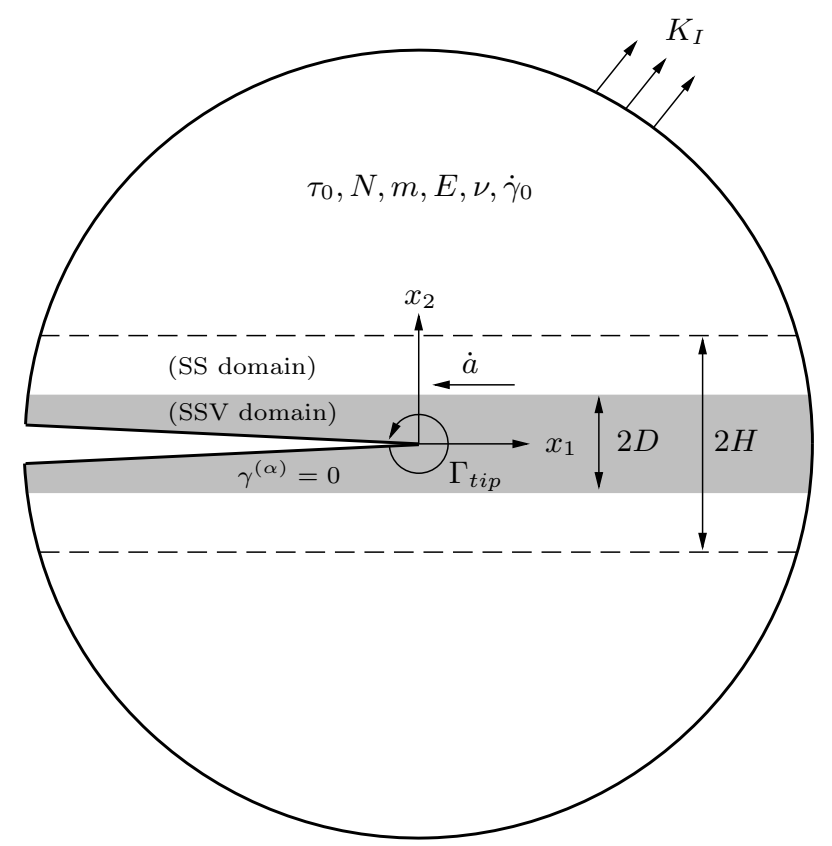

Figure 1: Mode I crack growth at steady-state in rate-sensitive crystal plastic material. The SSV domain provide an elastic strip embedded in the steady-state domain (SS domain). The crack is loaded with an elastic $K_{I}$ far field. 

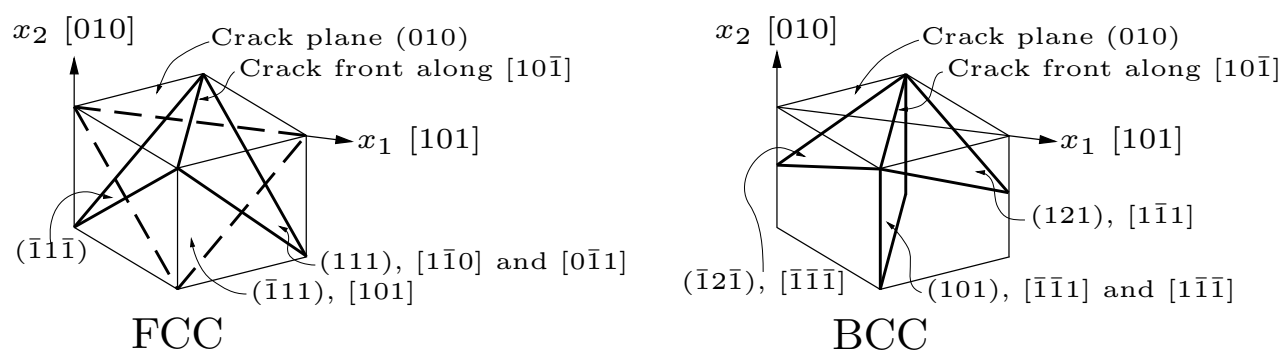

Figure 2: FCC and BCC crystal structure with the crack front along the $[10 \overline{1}]$ direction in the (010) crack plane (Rice, 1987). 

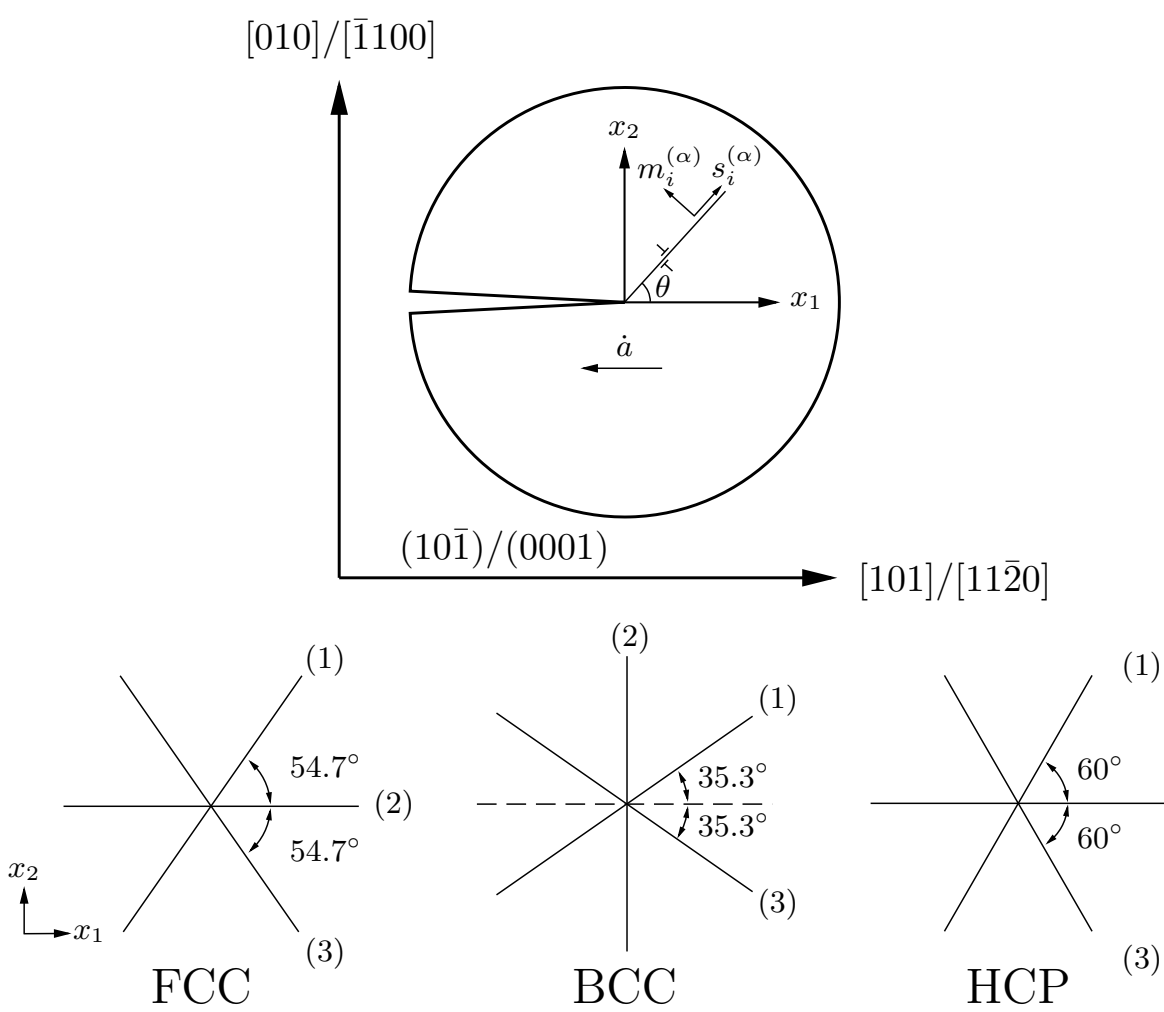

Figure 3: Mode I crack growth in single crystal with three and four Miller indices representing FCC/BCC and HCP, respectively. The sharp crack front is along the $[10 \overline{1}] /[0001]$ direction and the three effective slip systems are oriented as shown for FCC, BCC and HCP crystal structures. 


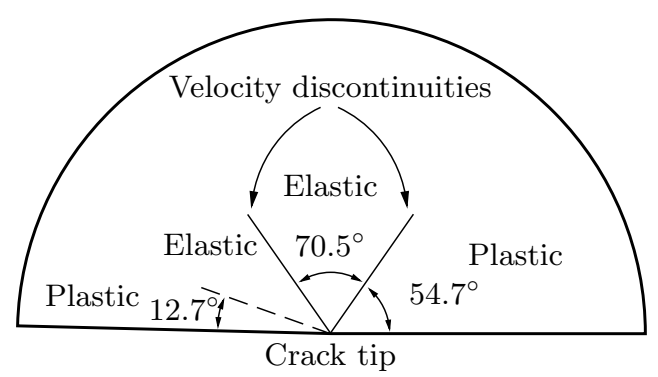

Figure 4: Velocity discontinuities and angle of secondary plastic zone at a steadily moving crack tip creating angular sectors for FCC and BCC crystals according to Rice (1987). 


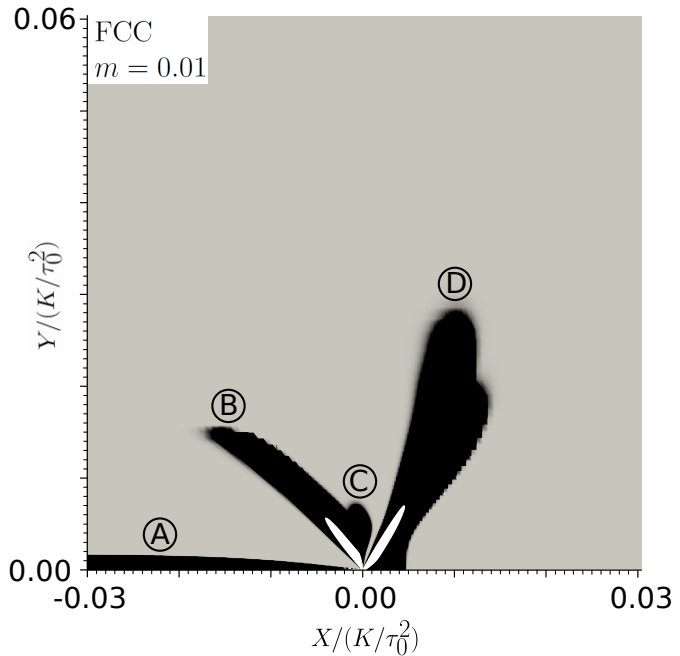

(a)

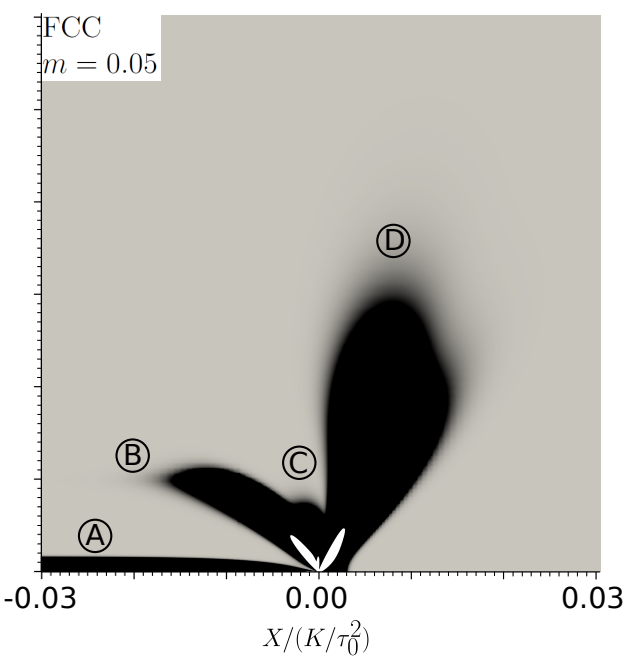

(b)

Figure 5: Accumulated slip rate, $\dot{\Lambda}$, for steady-state crack growth in perfectly plastic single crystal showing the plastic region, $\dot{\Lambda} G /\left(\zeta \dot{\gamma}_{0} \tau_{0}\right) \geq 1$, (black region) and a region of highly concentrated plastic straining, $\dot{\Lambda} G /\left(\zeta \dot{\gamma}_{0} \tau_{0}\right) \geq 2000$, (white region) in an FCC crystal for constant crack velocity $\zeta=1000$ with (a) $m=0.01$, and (b) $m=0.05$. 


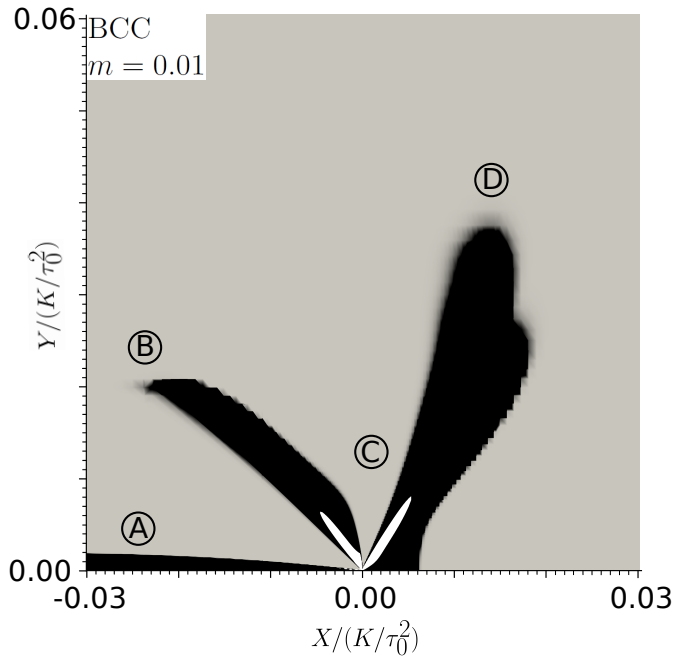

(a)

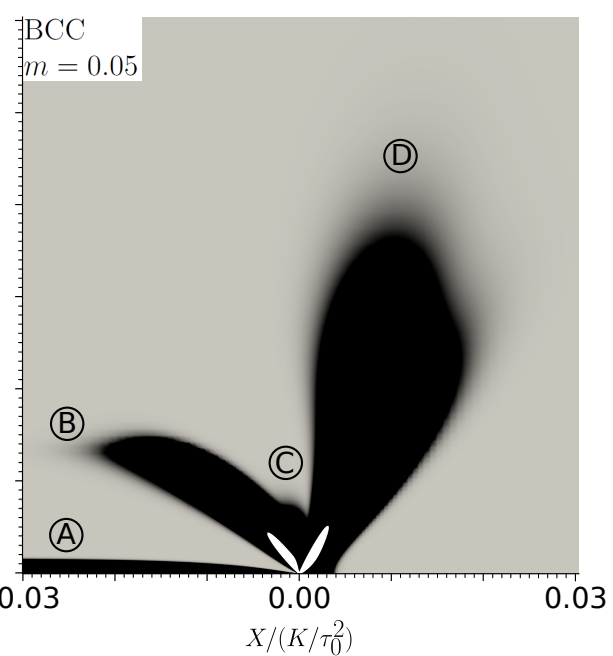

(b)

Figure 6: Accumulated slip rate, $\dot{\Lambda}$, for steady-state crack growth in perfectly plastic single crystal showing the plastic region, $\dot{\Lambda} G /\left(\zeta \dot{\gamma}_{0} \tau_{0}\right) \geq 1$, (black region) and a region of highly concentrated plastic straining, $\dot{\Lambda} G /\left(\zeta \dot{\gamma}_{0} \tau_{0}\right) \geq 2000$, (white region) in a BCC crystal for constant crack velocity $\zeta=1000$ with (a) $m=0.01$, and (b) $m=0.05$. 


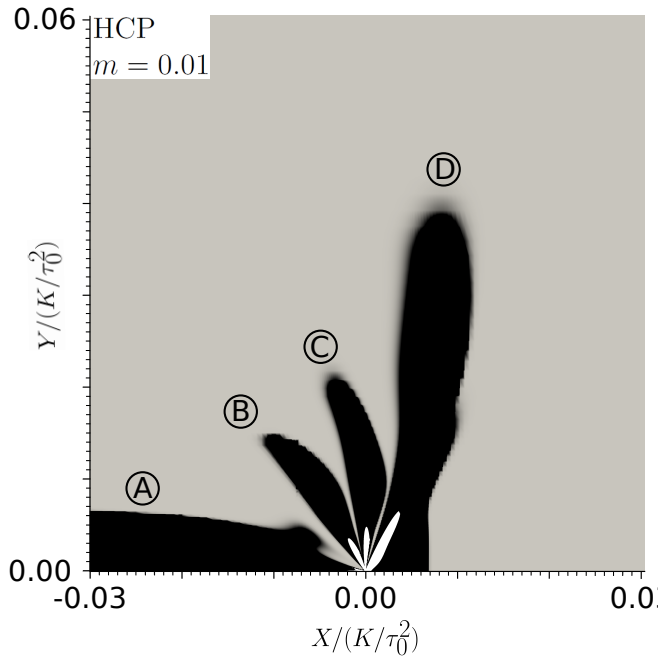

(a)

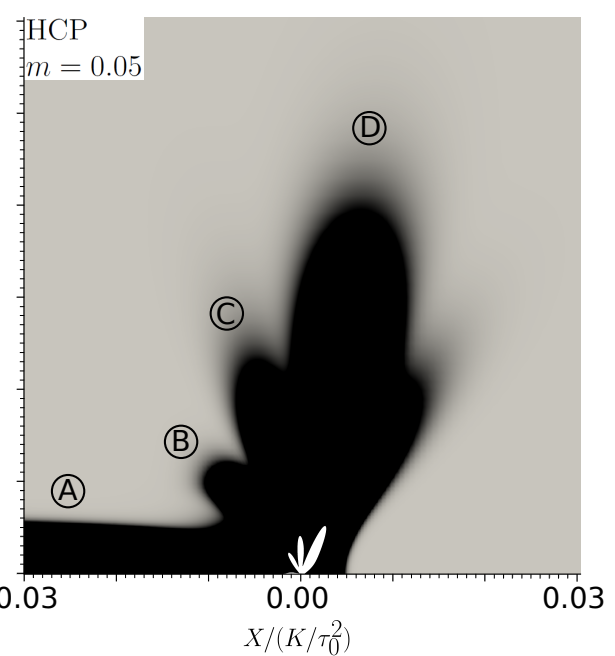

(b)

Figure 7: Accumulated slip rate, $\dot{\Lambda}$, for steady-state crack growth in perfectly plastic single crystal showing the plastic region, $\dot{\Lambda} G /\left(\zeta \dot{\gamma}_{0} \tau_{0}\right) \geq 1$, (black region) and a region of highly concentrated plastic straining, $\dot{\Lambda} G /\left(\zeta \dot{\gamma}_{0} \tau_{0}\right) \geq 2000$, (white region) in an HCP crystal for constant crack velocity $\zeta=1000$ with (a) $m=0.01$, and (b) $m=0.05$. 


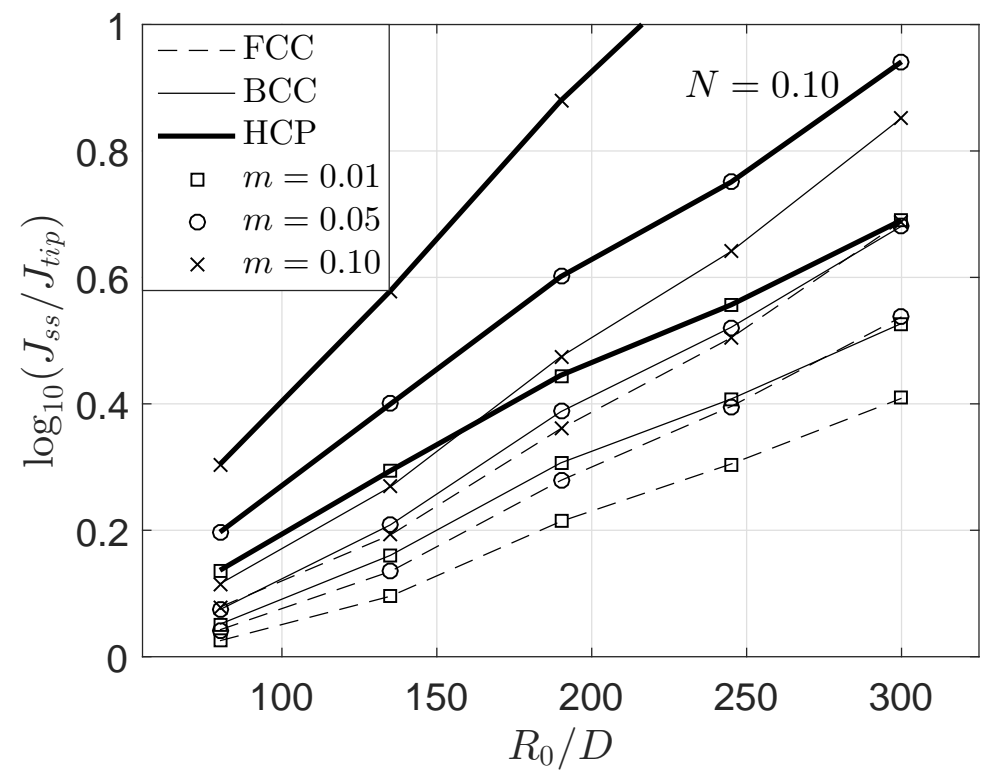

(a)

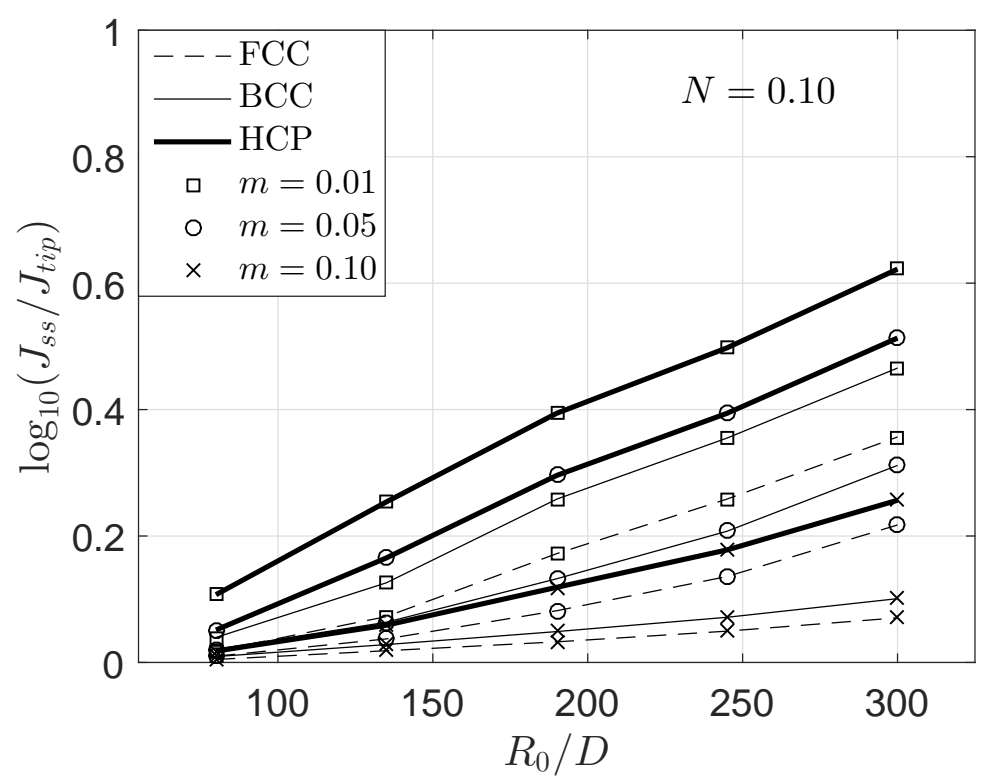

(b)

Figure 8: Crack tip shielding ratio vs. inverse of dislocation free region (SSV), $D$, for single crystal with parameters; $N=0.10$ and velocity (a) $\zeta=10$, and (b) $\zeta=1000$. 


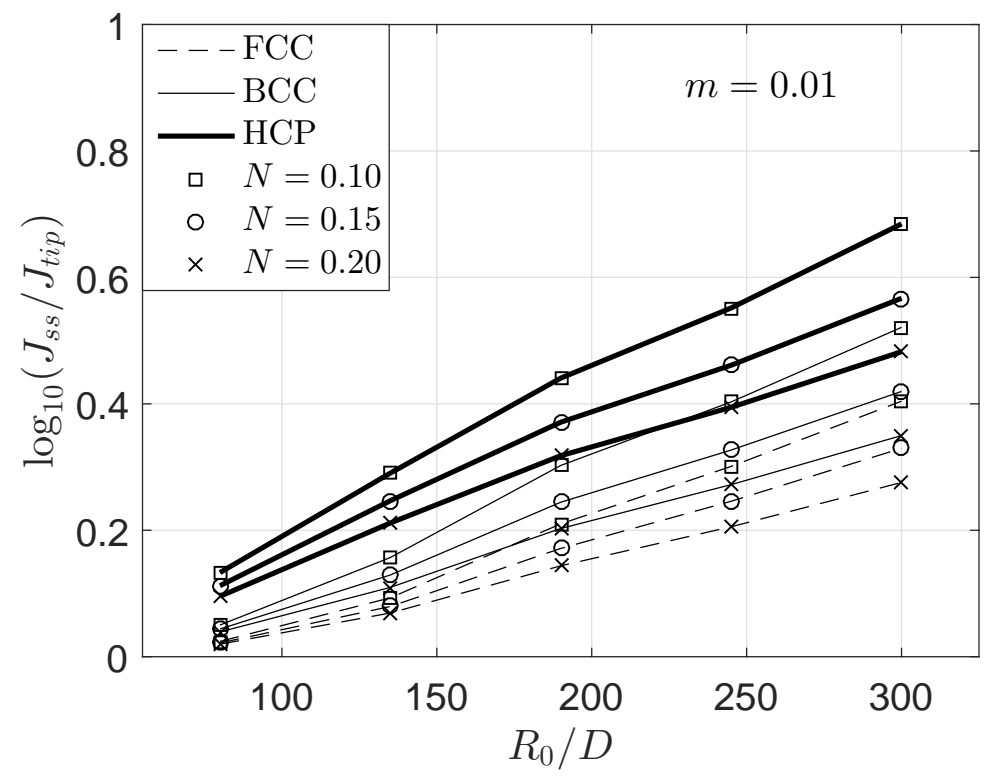

(a)

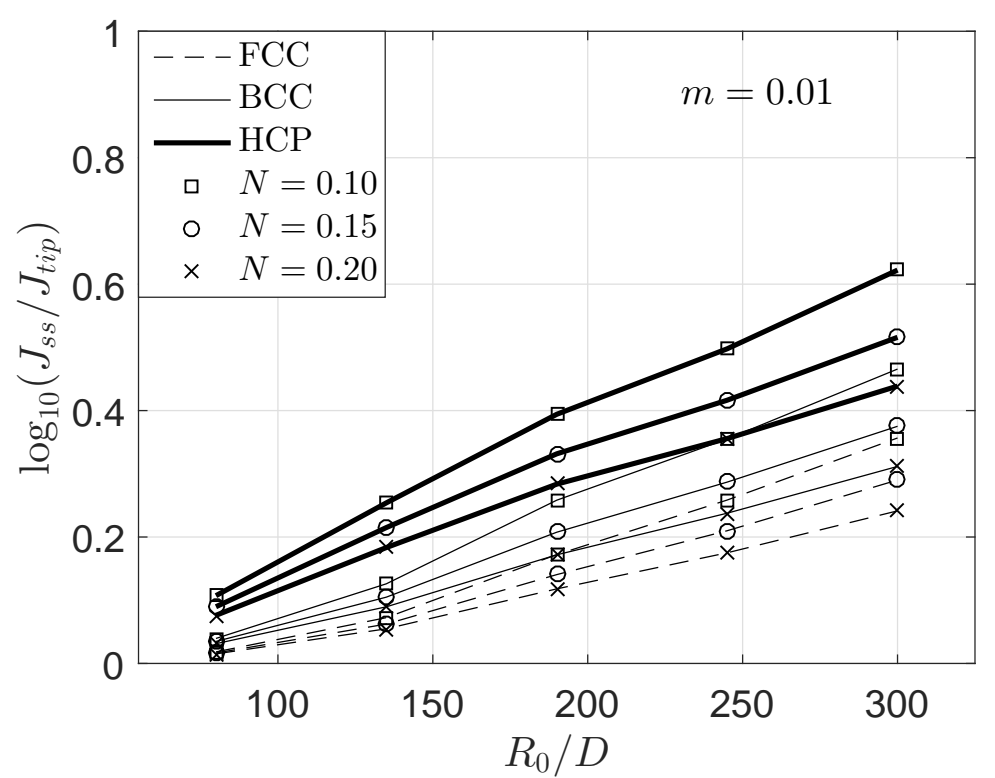

(b)

Figure 9: Crack tip shielding ratio vs. inverse of dislocation free region (SSV), $D$, for single crystal with parameters; $m=0.01$ and velocity (a) $\zeta=10$, and (b) $\zeta=1000$. 


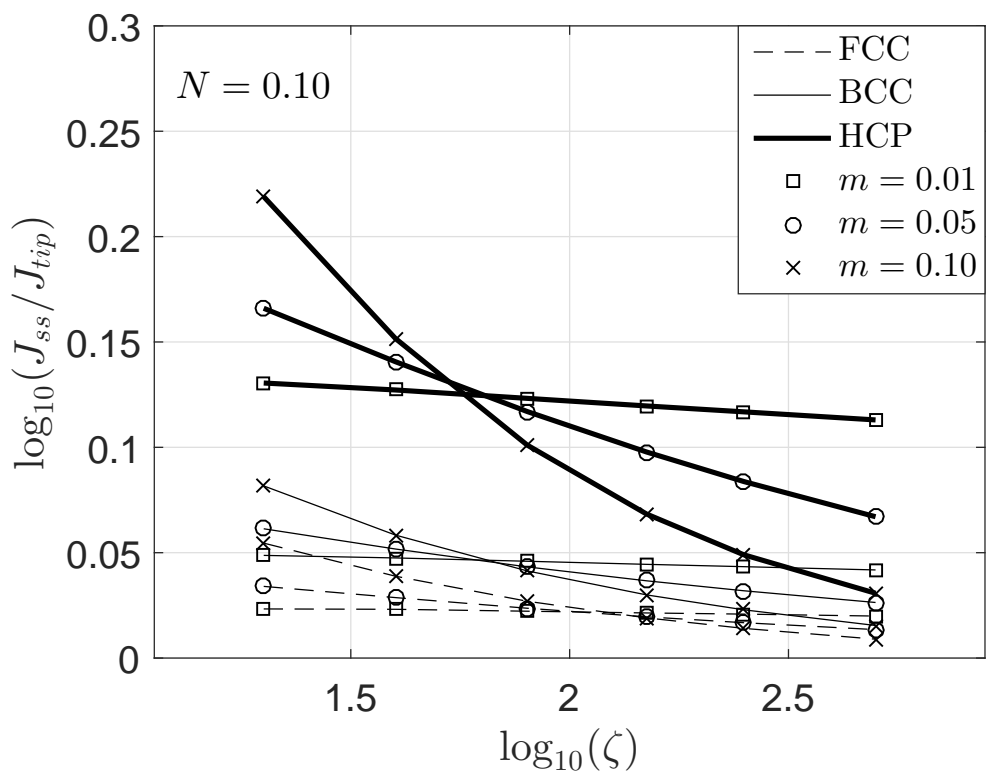

(a)

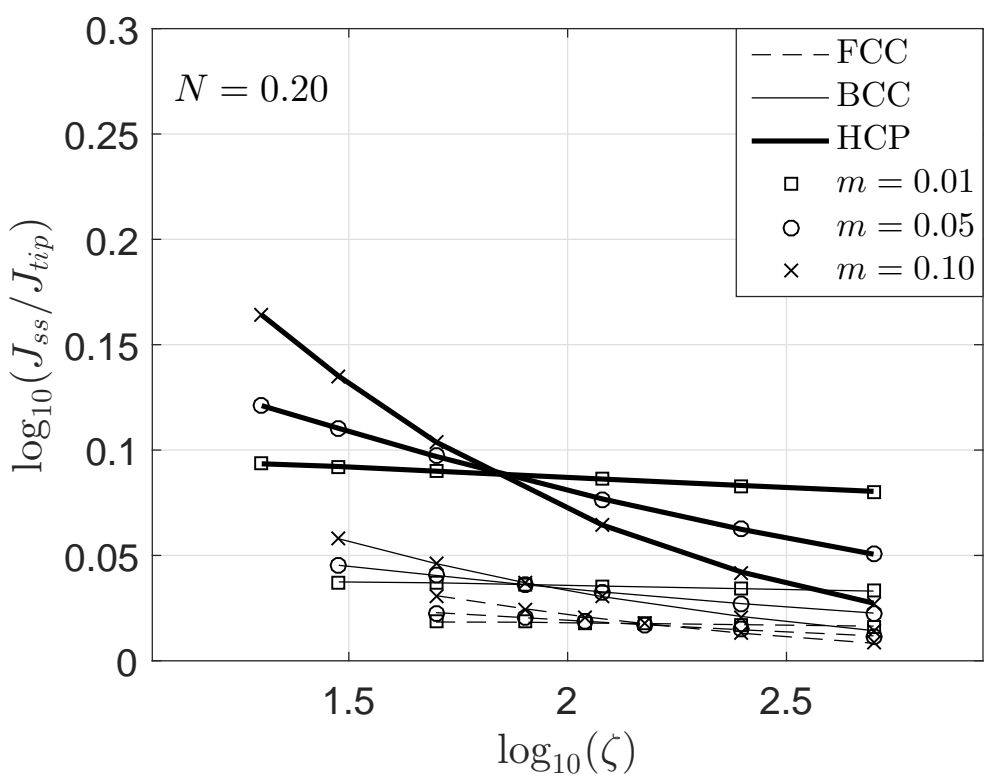

(b)

Figure 10: Crack tip shielding ratio as function of velocity for constant SSV domain size $R_{0} / D=80$ with hardening exponent (a) $N=0.10$, and (b) $N=0.20$. 
${ }_{597}$ List of Tables

${ }_{598} \quad 1 \quad$ Material properties. . . . . . . . . . . . . . . 33

${ }_{599} 2$ Crystallographic slip systems and the corresponding effective

$600 \quad$ slip systems. . . . . . . . . . . . . . . . . . . 34 


\begin{tabular}{cll}
\hline Parameter & Significance & Value \\
\hline$\tau_{0} / G$ & Yield strain & 0.001 \\
$\nu$ & Poisson ratio & 0.3 \\
$N$ & Strain hardening exponent & $0-0.2$ \\
$m$ & Strain rate-sensitivity exponent & $0.01-0.1$ \\
$\dot{\gamma}_{0}$ & Reference slip rate & 0.002 \\
$\Gamma_{t i p}$ & Near tip fracture energy & $1 \mathrm{~J} / \mathrm{m}^{2}$ \\
\hline
\end{tabular}

Table 1: Material properties. 


\begin{tabular}{|c|c|c|c|}
\hline Effective slip system no. & $(1)$ & $(2)$ & $(3)$ \\
\hline \multicolumn{4}{|c|}{ FCC crystal } \\
\hline Angle to $[101]$ in $(\overline{1} 01)$ plane & $54.7^{\circ}$ & $0^{\circ}$ & $-54.7^{\circ}$ \\
\hline Crystallographic slip system (a) & $(111)[1 \overline{1} 0]$ & $(11 \overline{1})[101]$ & $(\overline{1} 1 \overline{1})[0 \overline{1} \overline{1}]$ \\
\hline Crystallographic slip system (b) & $(111)[0 \overline{1} 1]$ & $(\overline{1} 11)[101]$ & $(\overline{1} 1 \overline{1})[\overline{1} \overline{1} 0]$ \\
\hline$\beta^{(\alpha)}=\frac{s_{i}^{(\alpha a)} m_{j}^{(\alpha a)}+s_{i}^{(\alpha b)} m_{j}^{(\alpha b)}}{s_{i}^{(\alpha)} m_{j}^{(\alpha)}}$ & $\sqrt{3}$ & $\frac{2}{\sqrt{3}}$ & $\sqrt{3}$ \\
\hline$\lambda^{(\alpha)}=\frac{\tau^{(\alpha)}}{\tau^{(\alpha a)}}=\frac{\tau^{(\alpha)}}{\tau^{(\alpha b)}}$ & $\frac{2}{\sqrt{3}}$ & $\sqrt{3}$ & $\frac{2}{\sqrt{3}}$ \\
\hline \multicolumn{4}{|c|}{ BCC crystal } \\
\hline Angle to $[101]$ in $(\overline{101})$ plane $\left[^{\circ}\right]$ & $35.3^{\circ}$ & $90^{\circ}$ & $-35.3^{\circ}$ \\
\hline Crystallographic slip system (a) & $(121)[1 \overline{1} 1]$ & $(101)[\overline{1} \overline{1} 1]$ & $(\overline{1} 2 \overline{1})[\overline{1} \overline{1} \overline{1}]$ \\
\hline Crystallographic slip system (b) & - & $(101)[1 \overline{1} \overline{1}]$ & - \\
\hline$\beta^{(\alpha)}=\frac{s_{i}^{(\alpha a)} m_{j}^{(\alpha a)}+s_{i}^{(\alpha b)} m_{j}^{(\alpha b)}}{s_{i}^{(\alpha)} m_{j}^{(\alpha)}}$ & 1 & $\frac{2}{\sqrt{3}}$ & 1 \\
\hline$\lambda^{(\alpha)}=\frac{\tau^{(\alpha)}}{\tau^{(\alpha a)}}=\frac{\tau^{(\alpha)}}{\tau^{(\alpha b)}}$ & 1 & $\sqrt{3}$ & 1 \\
\hline \multicolumn{4}{|c|}{ HCP crystal } \\
\hline Angle to $[11 \overline{2} 0]$ in $(0001)$ plane $\left[^{\circ}\right]$ & $60^{\circ}$ & $0^{\circ}$ & $-60^{\circ}$ \\
\hline Crystallographic slip system (a) & $(10 \overline{1} 0)[1 \overline{2} 10]$ & $(1 \overline{1} 00)[\overline{1} \overline{1} 20]$ & $(01 \overline{1} 0)[2 \overline{1} \overline{1} 0]$ \\
\hline Crystallographic slip system (b) & - & - & - \\
\hline$\beta^{(\alpha)}=\frac{s_{i}^{(\alpha a)} m_{j}^{(\alpha a)}+s_{i}^{(\alpha b)} m_{j}^{(\alpha b)}}{s_{i}^{(\alpha)} m_{j}^{(\alpha)}}$ & 1 & 1 & 1 \\
\hline$\lambda^{(\alpha)}=\frac{\tau^{(\alpha)}}{\tau^{(\alpha a)}}=\frac{\tau^{(\alpha)}}{\tau^{(\alpha b)}}$ & 1 & 1 & 1 \\
\hline
\end{tabular}

Table 2: Crystallographic slip systems and the corresponding effective slip systems. 